\title{
The well-posedness of stochastic Kawahara equation: fixed point argument and Fourier restriction method
}

\author{
Abd-Allah Hyder ${ }^{1,2^{*}}$ (D) and M. Zakarya ${ }^{1,3}$
}

\section{${ }^{*}$ Correspondence:}

abdallah.hyder@yahoo.com

'Department of Mathematics,

College of Science, King Khalid

University, P.O. Box 9004 Abha,

Saudi Arabia

Full list of author information is

available at the end of the article

\begin{abstract}
In this paper, we investigate the Cauchy problem for the stochastic Kawahara equation, which is a fifth-order shallow water wave equation. We prove local well-posedness for data in $H^{s}(\mathbb{R}), s>-7 / 4$. Moreover, we get global existence for $L^{2}(\mathbb{R})$ solutions. Due to the non-zero singularity of the phase function, a fixed point argument and Fourier restriction method are proposed.
\end{abstract}

Keywords: Kawahara equation, Well-posedness, Wiener process, Fixed point theorem, Fourier restriction method

AMS Subject Classification: $60 \mathrm{H} 15,49 \mathrm{~K} 40,60 \mathrm{H} 40$

\section{Introduction}

In this paper, we consider the Cauchy problem for the stochastic Kawahara equation:

$$
u_{t}+\alpha u_{5 x}+\beta u_{3 x}+\gamma u_{x}+\mu u u_{x}=\Phi \frac{\partial^{2} B}{\partial t \partial x},
$$

where $\alpha \neq 0, \beta$, and $\gamma$ are real numbers; $\mu$ is a complex number; $u$ is a stochastic process defined on $(x, t) \in \mathbb{R} \times \mathbb{R}_{+} ; \Phi$ is a linear operator; and $B$ is a two-parameter Brownian motion on $\mathbb{R} \times \mathbb{R}_{+}$, that is, a zero mean Gaussian process whose correlation function is given by:

$$
\mathbb{E}(B(x, t) B(y, s))=(x \wedge y)(t \wedge s), \quad t, s \geq 0, x, y \in \mathbb{R} .
$$

In general, the covariance operator $\Phi$ can be described by a kernel $\mathcal{K}(x, y)$. The correlation function of the noise is then given by

$$
\mathbb{E}\left(\Phi \frac{\partial^{2} B}{\partial t \partial x}(x, t) \Phi \frac{\partial^{2} B}{\partial t \partial x}(y, s)\right)=c(x, y) \delta_{t-s},
$$

where $t, s \geq 0, x, y \in \mathbb{R}, \delta$ is the Dirac function and

$$
c(x, y)=\int_{\mathbb{R}} \mathcal{K}(x, z) \mathcal{K}(y, z) d z .
$$

Consider a fixed probability space $(\Omega, \mathcal{F}, P)$ adapted to a filtration $\left(\mathcal{F}_{t}\right)_{t \geq 0}$. As usual, we can rewrite the right hand side of Eq. (1) as the time derivative of a cylindrical Wiener process on $L^{2}(\mathbb{R})$ by setting:

(c) The Author(s). 2019 Open Access This article is distributed under the terms of the Creative Commons Attribution 4.0 International License (http://creativecommons.org/licenses/by/4.0/), which permits unrestricted use, distribution, and reproduction in any medium, provided you give appropriate credit to the original author(s) and the source, provide a link to the Creative Commons license, and indicate if changes were made. 


$$
W(t)=\frac{\partial B}{\partial x}=\sum_{i \in \mathbb{N}} \beta_{i}(t) e_{i}
$$

where $\left(e_{i}\right)_{i \in \mathbb{N}}$ is an orthonormal basis of $L^{2}(\mathbb{R})$ and $\left(\beta_{i}\right)_{i \in \mathbb{N}}$ is a sequence of mutually independent real Brownian motions in $(\Omega, \mathcal{F}, P)$. Let us rewrite Eq. (1) in its Itô form as follows:

$$
\left\{\begin{array}{l}
d u+\left(\alpha u_{5 x}+\beta u_{3 x}+\gamma u_{x}+\mu u u_{x}\right) d t=\Phi d W(t) \\
u(x, 0)=u_{0}(x)
\end{array}\right.
$$

In order to obtain local well-posedness of Eq. (1), we mainly work on the general mild formulation of Cauchy problem (4) as below:

$$
u(t)=U(t) u_{0}+\int_{0}^{t} U(t-s)\left(\mu u u_{x}\right) d s+\int_{0}^{t} U(t-s) \Phi d W(s) .
$$

Here, $U(t)=\mathfrak{F}_{x}^{-1} \exp (-i t \phi(\xi)) \mathfrak{F}_{x}$ is the unitary group of operators related to the linearized equation:

$$
u_{t}+\alpha u_{5 x}+\beta u_{3 x}+\gamma u_{x}=0, \quad(x, t) \in \mathbb{R} \times \mathbb{R}_{+},
$$

where $\phi(\xi)=\alpha \xi^{5}-\beta \xi^{3}+\gamma \xi$ is the phase function and $\mathfrak{F}_{x}$ (or " $\hat{\imath}$ ") is the usual Fourier transform in the $x$ variable. We note that the phase function $\phi$ has non-zero singularity. This differs from the phase function of the linear Korteweg-de Vries (KdV) equation (see [1]) and causes some difficulties in the problem. To avoid these difficulties, we eliminate the singularity of the phase function $\phi$ by using the Fourier restriction operators [2]:

$$
P^{N} f=\int_{|\xi| \geq N} e^{i x \xi} \hat{f}(\xi) d \xi, \quad P_{N} f=\int_{|\xi| \leq N} e^{i x \xi \hat{f}}(\xi) d \xi, \quad \forall N>0 .
$$

In the case of $\Phi \equiv 0$ (effect of the noise does not exist), Eq. (1) is reduced to the deterministic Kawahara equation:

$$
u_{t}+\alpha u_{5 x}+\beta u_{3 x}+\gamma u_{x}+\mu u u_{x}=0, \quad(x, t) \in \mathbb{R} \times \mathbb{R}_{+} .
$$

As aforesaid in [3-5], Eq. (7) is a fifth-order shallow water wave equation. It arises in study of the water waves with surface tension, in which the Bond number takes on the critical value, where the Bond number represents a dimensionless magnitude of surface tension in the shallow water regime. If we consider a realistic situation, in which a nonconstant pressure affects on the surface of the fluid or the bottom of the layer is not flat, it is meaningful to add a forcing term to Eq. (7). This term can be given by the gradient of the exterior pressure or of the function whose graph defines the bottom [6, 7]. This paper focuses on the case when the forcing term is of additive white noise type. This leads us to study the stochastic fifth-order shallow water wave Eq. (1). By means of white noise functional analysis, the analytical white noise functional solutions for the nonlinear stochastic partial differential equations (SPDEs) can be investigated. This subject is attracting more and more attention [8-15].

It is well known that the Cauchy problem (4) is locally well-posed for data in $H^{s}(\mathbb{R}), s \in$ $\mathbb{R}$, if for any finite time $T$, there exists a locally continuous mapping that transfers $u_{0} \in$ 
$H^{s}(\mathbb{R})$ to a unique solution $u \in C\left([0, T] ; H^{s}(\mathbb{R})\right)$. If the solution mapping exists for all time, we say that the Cauchy problem (4) is globally well-posed [16].

In [17], Huo obtained a local well-posedness result in $H^{s}(\mathbb{R})(s>-11 / 8)$ for the Kawahara equation. Moreover, Jia and Huo [18] proved the local well-posedness of the Kawahara and modified Kawahara equations for data in $H^{s}(\mathbb{R})$ with $s>-7 / 4$ and $s \geq-1 / 4$ respectively. The first well-posedness result for the Kaup-Kupershmidt equations was presented by Tao and Cui [19]. They proved that their Cauchy problems are locally wellposed in $H^{s}(\mathbb{R})$ for $s>5 / 4$ and $s>301 / 108$, respectively. Thereafter, Zhao and Gu [20] lowered the regularity of the initial data space to $s>9 / 8$ and improved the preceding result in [19]. Also, using a Fourier restriction method, a local well-posedness result for the Kaup-Kupershmidt equations was established in [18] for data in $H^{s}(\mathbb{R})$ with $s>0$ and $s>-1 / 4$, respectively.

If $\alpha=\gamma=0$, the model (7) is minified to the famous $\mathrm{KdV}$ equation:

$$
u_{t}+\beta u_{3 x}+\mu u u_{x}=0, \quad(x, t) \in \mathbb{R} \times \mathbb{R}_{+} .
$$

The well-posedness of Eq. (8) was studied by Kenig, Ponce, and Vega [21]. They proved that its Cauchy problem is locally well-posed in $H^{s}(\mathbb{R})$ for $s>-3 / 4$. Also, Ponce [1] discussed the general fifth-order shallow water wave equation:

$$
u_{t}+u_{x}+c_{1} u u_{x}+c_{2} u_{3 x}+c_{3} u_{x} u_{x x}+c_{4} u u_{3 x}+c_{5} u_{5 x}=0 \quad(x, t) \in \mathbb{R} \times \mathbb{R}_{+}
$$

and gave a global well-posedness result of its Cauchy problem for data in $H^{4}(\mathbb{R})$. The wellposedness of the SPDEs has been the subject of a large amount of work. de Bouard and Debussche [22] considered the stochastic KdV equation forced by a random term of white noise type. They proved existence and uniqueness of solutions in $H^{1}(\mathbb{R})$ and existence of martingale solutions in $L^{2}(\mathbb{R})$ in the case of additive and multiplicative noise, respectively. Since that time, many researchers paid more attention to investigate the Cauchy problems for some SPDEs and have obtained a number of local and global well-posedness results [23-25].

The goal of this paper is to investigate the Cauchy problem of the stochastic Kawahara Eq. (1), where the random force is of additive white noise type. By employing a Fourier restriction method, a Banach fixed point theorem, and some basic inequalities, we show that Eq. (1) is locally well-posed for data in $H^{s}(\mathbb{R}), s>-7 / 4$. Also, we give global existence for $L^{2}(\mathbb{R})$ solutions. An outline of this paper is as follows. The "Main results" section contains precise statement of our new results and some important function spaces. In the section "The stochastic convolution estimate", we give an estimation of the stochastic convolution term via a Fourier restriction method and some basic inequalities. In the section "Local well-posedness: proof of Theorem 1", we use the stochastic estimation proved in the section "The stochastic convolution estimate" and the Banach fixed point theorem to obtain a local well-posedness result of Eq. (1). In the section "Global well-posedness: proof of Theorem 2", we extend our technique and show global well-posedness result of Eq. (1). The "Summary and discussion" section is devoted to the summary and discussion.

\section{Main results}

Before giving the precise statement of our main results, we introduce some notations and assumptions. 
Definition 1 For $s, b \in \mathbb{R}$ the space $\mathfrak{X}_{s, b}$ is defined to be the completion of the Schwartz function space $\mathcal{S}\left(\mathbb{R}^{2}\right)$ with respect to the norm:

$$
\|u\|_{\mathfrak{X}_{s, b}}=\|U(-t) u\|_{H_{x}^{s} H_{t}^{b}}=\left\|\langle\xi\rangle^{s}\langle\tau+\phi(\xi)\rangle^{b} \mathfrak{F} u\right\|_{L_{\xi}^{2} L_{\tau}^{2}},
$$

where $\langle\cdot\rangle=1+|\cdot|$.

Definition 2 For $T>0, \mathfrak{X}_{s, b}^{T}$ is the space of restrictions to $[0, T]$ of functions in $\mathfrak{X}_{s, b}$ endowed with the norm:

$$
\|u\|_{\mathfrak{X}_{s, b}^{T}}=\inf \left\{\|\tilde{u}\|_{\mathfrak{X}_{s, b}}: \tilde{u} \in \mathfrak{X}_{s, b}, u=\left.\tilde{u}\right|_{[0, T]}\right\}
$$

Theorem 1 Assume that $s>-\frac{7}{4}, \Phi \in L_{2}^{0, s}, b \in\left(0, \frac{1}{2}\right)$ and $b$ is close enough to $\frac{1}{2}$. If $u_{0} \in H^{s}(\mathbb{R})$ for almost surely $\omega \in \Omega$ and $u_{0}$ is $\mathcal{F}_{0}-$ measurable. Then for almost surely $\omega \in \Omega$, there exists a constant $T_{\omega}>0$ and a unique solution $u$ of the Cauchy problem (4) on $\left[0, T_{\omega}\right]$ which satisfies:

$$
u \in C\left(\left[0, T_{\omega}\right] ; H^{s}(\mathbb{R})\right) \cap \mathfrak{X}_{s, b}^{T_{\omega}} .
$$

In fact the $L^{2}$-norm is preserved for a solution of the Kawahara equation [4]. Therefore, in the case of $s=0$, we can obtain a global existence result for Eq. (1). Precisely, we have:

Theorem 2 Let $u_{0} \in L^{2}\left(\Omega, L^{2}(\mathbb{R})\right)$ be an $\mathcal{F}_{0}$-measurable initial data, and let $\Phi \in L_{2}^{0,0}$. Then, the solution $u$ given by Theorem 1 is global and satisfies:

$$
u \in L^{2}\left(\Omega ; C\left(\left[0, T_{0}\right] ; H^{s}(\mathbb{R})\right)\right), \text { for any } T_{0}>0 .
$$

\section{The stochastic convolution estimate}

In this section, using the Fourier restriction method, the properties of Itô stochastic integral and some basic inequalities, we give an estimation for the last term in Eq. (5), which is the stochastic convolution:

$$
u_{l}(t):=\int_{0}^{t} U(t-s) \Phi d W(s) .
$$

Choose $\chi \in C_{0}^{\infty}\left(\mathbb{R}_{+}\right)$such that $\chi(t)=0$ for $t>0, \chi(t)=1$ for $0<t<1$ and $\chi(t)=0$ for $t \geq 2$. Hence, $\chi \in H^{b}(\mathbb{R})$ for any $b<\frac{1}{2}$. Let $H_{t}^{b}:=H^{b}([0, T] ; \mathbb{R})$ be the Sobolev space in the time variable $t$ with the norm:

$$
\|\psi\|_{H_{t}^{b}}^{2}:=\|\psi\|_{L^{2}(\mathbb{R})}^{2}+\int_{\mathbb{R}} \int_{\mathbb{R}} \frac{\left|\psi\left(t_{1}\right)-\psi\left(t_{2}\right)\right|^{2}}{\left|t_{1}-t_{2}\right|^{1+2 b}} d t_{1} d t_{2}, \quad \psi \in H_{t}^{b} .
$$

Now, we state and prove the estimation of the stochastic convolution (12) as follows:

Lemma 1 Assume that $s, b \in \mathbb{R}$ with $b \in\left(0, \frac{1}{2}\right)$, and let $\Phi \in L_{2}^{0, s}$ Then, $u_{l}$ defined by (12) satisfies:

$$
\chi u_{l} \in L^{2}\left(\Omega, \mathfrak{X}_{s, b}\right)
$$

and 


$$
\mathbb{E}\left(\left\|\chi u_{l}\right\|_{\mathfrak{X}_{s, b}}^{2}\right) \leq N(b, \chi)\|\Phi\|_{L_{0}^{0, s}}^{2}
$$

where $N(b, \chi)$ is a constant that depends on $b,\|\chi\|_{H_{t}^{b}},\left\||t|^{\frac{1}{2}} \chi\right\|_{L_{t}^{2}}$ and $\left\||t|^{\frac{1}{2}} \chi\right\|_{L_{t}^{\infty}}$,

Proof Let us introduce the function

$$
w(t, .)=\chi(t) \int_{0}^{t} U(-s) \Phi d W(s), \quad t \in \mathbb{R}_{+} .
$$

This implies that $U(t) w(t,)=.\chi(t) u_{l}(t)$. Thus, by Eq. (10), we have:

$$
\begin{aligned}
\mathbb{E}\left(\left\|\chi u_{l}\right\|_{\mathfrak{X}_{s, b}}^{2}\right) & =\mathbb{E}\left(\int_{\mathbb{R}} \int_{\mathbb{R}}(1+|\xi|)^{2 s}(1+|\tau|)^{2 b}\left|\mathfrak{F}_{x} w(t, \xi)\right|^{2} d \tau d \xi\right) \\
& =\int_{\mathbb{R}}(1+|\xi|)^{2 s} \mathbb{E}\left(\left\|\mathfrak{F}_{x} w(., \xi)\right\|_{H_{t}^{b}}^{2}\right) d \xi
\end{aligned}
$$

According to the expansion (3) of the cylindrical Wiener process and Eq. (13), we have:

$$
\mathbb{E}\left(\left\|\mathfrak{F}_{x} w(., \xi)\right\|_{H_{t}^{b}}^{2}\right)=S_{1}+S_{2}
$$

where,

$$
\begin{aligned}
& S_{1}=\sum_{i \in \mathbb{N}}\left|\hat{\Phi e_{i}}\right|^{2}\left[\mathbb{E}\left(\left\|\chi(t) \int_{0}^{t} e^{i s \phi(\xi)} d \beta_{i}(s)\right\|_{L^{2}(\mathbb{R})}^{2}\right)\right] \\
& S_{2}=\sum_{i \in \mathbb{N}}\left|\hat{\Phi e_{i}}\right|^{2}\left[\mathbb{E}\left(\int_{\mathbb{R}} \int_{\mathbb{R}} \frac{\left|\begin{array}{c}
\chi\left(t_{1}\right) \int_{0}^{t_{1}} e^{i s \phi(\xi)} d \beta_{i}(s) \\
-\chi\left(t_{2}\right) \int_{0}^{t_{2}} e^{i s \phi(\xi)} d \beta_{i}(s)
\end{array}\right|}{\left|t_{1}-t_{2}\right|^{1+2 b}} d t_{1} d t_{2}\right)\right] .
\end{aligned}
$$

From the Itô isometry formula, we get:

$$
\begin{aligned}
S_{1} & =\sum_{i \in \mathbb{N}}\left|\hat{\Phi e_{i}}\right|^{2} \int_{0}^{2}|\chi(t)|^{2} \mathbb{E}\left(\left|\int_{0}^{t} e^{i s \phi(\xi)} d \beta_{i}(s)\right|^{2}\right) d t \\
& =\left\||t|^{\frac{1}{2}} \chi\right\|_{L_{t}^{2}}^{2} \sum_{i \in \mathbb{N}}\left|\hat{\Phi e_{i}}\right|^{2} .
\end{aligned}
$$

To estimate $S_{2}$, we have: 


$$
\begin{aligned}
& S_{2}=\sum_{i \in \mathbb{N}}\left|\hat{\Phi e_{i}}\right|^{2}\left[\mathbb{E}\left(\int_{\mathbb{R}} \int_{\mathbb{R}} \frac{\left|\begin{array}{c}
\chi\left(t_{1}\right) \int_{0}^{t_{1}} e^{i s \phi(\xi)} d \beta_{i}(s) \\
-\chi\left(t_{2}\right) \int_{0}^{t_{2}} e^{i s \phi(\xi)} d \beta_{i}(s)
\end{array}\right|}{\left|t_{1}-t_{2}\right|^{1+2 b}} d t_{1} d t_{2}\right)\right] \\
& =2 \sum_{i \in \mathbb{N}}\left|\hat{\Phi} e_{i}\right|^{2} \int_{t_{2}>0} \int_{t_{1}<t_{2}} \frac{\mathbb{E}\left(\left|\begin{array}{c}
\chi\left(t_{1}\right) \int_{0}^{t_{1}} e^{i s \phi(\xi)} d \beta_{i}(s) \\
-\chi\left(t_{2}\right) \int_{0}^{t_{2}} e^{i s \phi(\xi)} d \beta_{i}(s)
\end{array}\right|^{2}\right)}{\left|t_{1}-t_{2}\right|^{1+2 b}} d t_{1} d t_{2} \\
& \leq \sum_{i \in \mathbb{N}}\left|\hat{\Phi} \hat{e}_{i}\right|^{2}\left[2 \int_{t_{2}>0} \int_{t_{1}<0} \frac{\left|\chi\left(t_{2}\right)\right|^{2} \mathbb{E}\left(\left|\int_{0}^{t_{2}} e^{i s \phi(\xi)} d \beta_{i}(s)\right|^{2}\right)}{\left|t_{1}-t_{2}\right|^{1+2 b}} d t_{1} d t_{2}\right. \\
& \left.+2 \int_{t_{2}>0} \int_{0<t_{1}<t_{2}} \frac{\mathbb{E}\left(\left|\begin{array}{c}
\chi\left(t_{1}\right) \int_{0}^{t_{1}} e^{i s \phi(\xi)} d \beta_{i}(s) \\
-\chi\left(t_{2}\right) \int_{0}^{t_{1}} e^{i s \phi(\xi)} d \beta_{i}(s) \\
+\chi\left(t_{2}\right) \int_{t_{1}}^{t_{2}} e^{i s \phi(\xi)} d \beta_{i}(s)
\end{array}\right|\right)}{\left|t_{1}-t_{2}\right|^{1+2 b}} d t_{1} d t_{2}\right] \\
& \leq \sum_{i \in \mathbb{N}}\left|\hat{\Phi} e_{i}\right|^{2}\left[2 \int_{t_{2}>0} \int_{t_{1}<0} \frac{\left|\chi\left(t_{2}\right)\right|^{2} \mathbb{E}\left(\left|\int_{0}^{t_{2}} e^{i s \phi(\xi)} d \beta_{i}(s)\right|^{2}\right)}{\left|t_{1}-t_{2}\right|^{1+2 b}} d t_{1} d t_{2}\right. \\
& +4 \int_{t_{2}>0} \int_{0<t_{1}<t_{2}} \frac{\left|\chi\left(t_{1}\right)-\chi\left(t_{2}\right)\right|^{2} \mathbb{E}\left(\left|\int_{0}^{t_{1}} e^{i s \phi(\xi)} d \beta_{i}(s)\right|^{2}\right)}{\left|t_{1}-t_{2}\right|^{1+2 b}} d t_{1} d t_{2} \\
& \left.+4 \int_{t_{2}>0} \int_{0<t_{1}<t_{2}} \frac{\left|\chi\left(t_{2}\right)\right|^{2} \mathbb{E}\left(\left|\int_{t_{1}}^{t_{2}} e^{i s \phi(\xi)} d \beta_{i}(s)\right|^{2}\right)}{\left|t_{1}-t_{2}\right|^{1+2 b}} d t_{1} d t_{2}\right] \\
& =\sum_{i \in \mathbb{N}}\left|\hat{\Phi e_{i}}\right|^{2}\left[I_{1}+I_{2}+I_{3}\right] \text {. }
\end{aligned}
$$

Now, we limit $I_{1}, I_{2}$, and $I_{3}$ separately,

$$
I_{1} \leq 2 \int_{0}^{2} t_{1}\left|\chi\left(t_{2}\right)\right|^{2} \int_{t_{1}<0} \frac{1}{\left|t_{1}-t_{2}\right|^{1+2 b}} d t_{1} d t_{2} \leq M_{b}\left\||t|^{\frac{1}{2}-b} \chi\right\|_{L_{t}^{2}}^{2} .
$$

Using Eq. (15) and the assumption that $2 b \in(0,1)$, we have

$$
\begin{aligned}
I_{2} & \leq 4 \int_{0}^{\infty} \int_{0}^{t_{2}} \frac{t_{1}\left|\chi\left(t_{1}\right)-\chi\left(t_{2}\right)\right|^{2}}{\left\|t_{1}-t_{2}\right\|^{1+2 b}} d t_{1} d t_{2} \\
& \leq 4 \int_{0}^{2} \int_{0}^{t_{2}} \frac{t_{1}\left|\chi\left(t_{1}\right)-\chi\left(t_{2}\right)\right|^{2}}{\left\|t_{1}-t_{2}\right\|^{1+2 b}} d t_{1} d t_{2} \\
& +4 \int_{2}^{\infty} \int_{0}^{2} \frac{t_{1}\left|\chi\left(t_{1}\right)\right|^{2}}{\left\|t_{1}-t_{2}\right\|^{1+2 b}} d t_{1} d t_{2} \\
& \leq 8\|\chi\|_{H_{t}^{b}}^{2}+4\left\||t|^{\frac{1}{2}} \chi\right\|_{L_{t}^{\infty}}^{2} \int_{0}^{\infty} \int_{0}^{2} \frac{1}{\left|t_{1}-t_{2}\right|^{1+2 b}} d t_{1} d t_{2} \\
& \leq 8\|\chi\|_{H_{t}^{b}}^{2}+M_{b}\left\||t|^{\frac{1}{2}} \chi\right\|_{L_{t}^{\infty}}^{2}
\end{aligned}
$$

Similarly,

$$
I_{3} \leq 4 \int_{0}^{2} \int_{0}^{t_{2}} \frac{\left|\chi\left(t_{2}\right)\right|^{2}}{\left|t_{1}-t_{2}\right|^{2 b}} d t_{1} d t_{2} \leq M_{b}\left\||t|^{\frac{1}{2}-b} \chi\right\|_{L_{t}^{2}}^{2} .
$$

Combining (20)-(24) with (17), we get

$$
\mathbb{E}\left(\left\|\mathfrak{F}_{x} w(., \xi)\right\|_{H_{t}^{b}}^{2}\right) \leq N(b, \chi) \sum_{i \in \mathbb{N}}\left|\hat{\Phi e_{i}}\right|^{2}
$$


where $N(b, \chi)=M_{b}\left(\|\chi\|_{H_{t}^{b}}+\left\||t|^{\frac{1}{2}} \chi\right\|_{L_{t}^{2}}+\left\||t|^{\frac{1}{2}} \chi\right\|_{L_{t}^{\infty}}\right)$. Hence, the estimate (14) comes from (16) and (25).

\section{Local well-posedness: proof of Theorem 1}

According to the stochastic estimation proved in the above section and the Banach fixed point theorem, we deduce a local well-posedness result of Eq. (1). That is, this section is devoted to the proof of Theorem 1. Let $v(t)=U(t) u_{0}$ and $\bar{u}=u(t)-v(t)-u_{l}(t)$, then Eq. (5) is equivalent to

$$
\bar{u}(t)=\mathcal{A} \bar{u}(t):=\frac{1}{2} \int_{0}^{t} U(t-s) \frac{\partial}{\partial x}\left(\bar{u}^{2}+u_{l}^{2}+v^{2}+2\left(\bar{u} u_{l}+\bar{u} v+v u_{l}\right)\right)(s) d s .
$$

Therefore, the goal of this section becomes to prove that $\mathcal{A}$ is a contraction mapping in

$$
\mathfrak{Y}_{R}^{T}=\left\{\bar{u} \in \mathfrak{X}_{s, b}^{T}:\|\bar{u}\|_{\mathfrak{X}_{s, b}^{T}} \leq R\right\}, \quad R>0, \quad T>0,
$$

where $R$ and $T$ are sufficiently large and small, respectively. Before doing this, we recall some previous results on the linear and bilinear estimates.

Lemma 2 [23] Assume that $a>0, b<\frac{1}{2}$ and $b$ is close enough to $\frac{1}{2}$. For $s \in \mathbb{R}, u_{0} \in$ $H^{s}(\mathbb{R})$, and $f \in \mathfrak{X}_{s,-a}^{T}$, we have:

$$
\left\|\int_{0}^{t} U(t-\tau) f(\tau) d \tau\right\|_{\mathfrak{X}_{s, b}^{T}} \leq C T^{1-a-b}\|f\|_{\mathfrak{X}_{s, b}^{T}}
$$

and

$$
\|v\|_{\mathfrak{X}_{s, b}^{T}} \leq\left\|u_{0}\right\|_{H^{s}}
$$

Lemma 3 [18] Assume that $a>0, b<\frac{1}{2}$, and $b$ is close enough to $\frac{1}{2}$. For $b^{\prime}>\frac{1}{2}, s>-\frac{7}{4}$, and $u_{1}, u_{2} \in \mathcal{S}\left(\mathbb{R}^{2}\right)$, we have:

$$
\left\|\frac{\partial}{\partial x}\left(u_{1} u_{2}\right)\right\|_{\mathfrak{X}_{s,-a}} \leq C\left\|u_{1}\right\|_{\mathfrak{X}_{s, b}}\left\|u_{1}\right\|_{\mathfrak{X}_{s, b^{\prime}}}
$$

provided that the right hand side is finite.

According to Lemmas 1, 2, and 3, we obtain

$$
\|\mathcal{A} \bar{u}\|_{\mathfrak{X}_{s, b}^{T}} \leq C^{\prime} T^{1-a-b}\left(R^{2}+\left\|u_{l}\right\|_{\mathfrak{X}_{s, b}^{T}}+\left\|u_{0}\right\|_{H^{s}}\right) .
$$

Therefore, for $\bar{u}_{1}, \bar{u}_{2} \in \mathfrak{Y}_{R}^{T}$, we get

$$
\left\|\mathcal{A} \bar{u}_{1}-\mathcal{A} \bar{u}_{2}\right\|_{\mathfrak{X}_{s, b}^{T}} \leq C^{\prime} T^{1-a-b}\left(R^{2}+\left\|u_{l}\right\|_{\mathfrak{X}_{s, b}^{T}}+\left\|u_{0}\right\|_{H^{s}}\right)\left\|\bar{u}_{1}-\bar{u}_{2}\right\|_{\mathfrak{X}_{s, b}^{T}} .
$$

Now, define the stopping time $T_{\omega}$ by:

$$
T_{\omega}=\inf \left\{t>0: 4 C^{\prime} t^{1-a-b} R_{\omega}^{T} \geq 1\right\},
$$

where $R_{\omega}^{T}=\left\|u_{l}\right\|_{\mathfrak{X}_{s, b}^{T}}+\left\|u_{0}\right\|_{H^{s}}$. Then, $\mathcal{A}$ maps the ball with center zero and radius $R_{\omega}^{T}$ in $\mathfrak{X}_{s, b}^{T_{\omega}}$ into itself, and

$$
\left\|\mathcal{A} \overline{u_{1}}-\mathcal{A} \overline{u_{2}}\right\|_{\mathfrak{X}_{s, b}^{T_{\omega}}} \leq \frac{3}{4}\left\|\overline{u_{1}}-\overline{u_{2}}\right\|_{\mathfrak{X}_{s, b}^{T_{\omega}}} .
$$

From the fixed point theory, $\mathcal{A}$ has a unique fixed point, which is the solution of (5) in $\mathfrak{X}_{s, b}^{T_{\omega}}$. Observe that $u=v+\bar{u}+u_{l} \in \mathfrak{X}_{s, b^{\prime}}^{T_{\omega}}+\mathfrak{X}_{s, b}^{T_{\omega}}$. 
In the remaining part of this section, we complete the proof by showing that $u \in$ $C\left(\left[0, T_{\omega}\right], H^{s}(\mathbb{R})\right)$. Taking in attention that $b<\frac{1}{2}, b^{\prime}>\frac{1}{2}$. By virtue of the Sobolev imbedding theorem, we have $v \in C\left(\left[0, T_{\omega}\right], H^{s}(\mathbb{R})\right)$. Under the condition that $\Phi \in L_{2}^{0, s}$ and the fact that $U(t)$ is a unitary group in $H^{s}(\mathbb{R})$, an application of Theorem 6.10 in [16] implies that $u_{l} \in C\left(\left[0, T_{\omega}\right] ; H^{s}(\mathbb{R})\right)$.

Now, choose a cutoff function $\chi_{T} \in C_{0}^{\infty}(\mathbb{R})$ such that $\chi_{T}(t)=1$ on [0,2], supp $\chi_{T} \subset$ $[-1,2]$, and $\chi_{T}(t)=0$ on $(-\infty,-1] \cup[2, \infty)$. Denote $\chi_{q}()=.\chi\left(q^{-1}().\right)$ for some $q \in \mathbb{R}$. By Lemma 3, we have $\tilde{u} \tilde{u}_{x} \in \mathfrak{X}_{s,-a}$ for any prolongation $\tilde{u}$ of $u$ in $\mathfrak{X}_{s, c}+\mathfrak{X}_{s, b}$. Therefore,

$$
\left\|\chi_{T} \int_{0}^{t} U(t-s)\left(\tilde{u}(s) \tilde{u}_{x}(s)\right)\right\|_{\mathfrak{X}_{s, 1-a}} \leq C\left\|\tilde{u}(s) \tilde{u}_{x}(s)\right\|_{\mathfrak{X}_{s,-a}} .
$$

Since $1-a>\frac{1}{2}$, then $\tilde{u} \in \mathfrak{X}_{s, b} \subset C\left(\left[0, T_{\omega}\right] ; H^{s}(\mathbb{R})\right)$. This completes the proof of Theorem 1.

\section{Global well-posedness: proof of Theorem 2}

Fix $T_{0}>0$ and assume that $u_{0}$ satisfies the conditions of Theorem 1. In this section, we present a proof of Theorem 2, that is, we show that the solution $u$ can be extended to the whole interval $\left[0, T_{0}\right]$. Let $\left(\Phi_{n}\right)_{n \in \mathbb{N}}$ be a sequence in $L_{0}^{0,4}$ such that

$$
\lim _{n \rightarrow \infty} \Phi_{n}=\Phi \quad \text { in } L_{2}^{0,0} \text {. }
$$

and let $\left(u_{0, n}\right)_{n \in \mathbb{N}}$ be another sequence in $L^{2}\left(\Omega, H^{s}(\mathbb{R})\right)$ such that

$$
\lim _{n \rightarrow \infty} u_{0, n}=u_{0} \quad \text { in } L^{2}\left(\Omega, L^{2}(\mathbb{R})\right) .
$$

By using reasoning similar to that in [23], we can find a unique solution $u_{n}$ in $C\left(\left[0, T_{0}\right], H^{3}(\mathbb{R})\right)$ for

$$
u_{n}=U(t) u_{0, n}+\int_{0}^{t} U(t-s)\left(u_{n}(s) \frac{\partial u_{n}}{\partial x}(s)\right) d s+\int_{0}^{t} U(t-s) \Phi_{n} d W(s) .
$$

By using the Itô formula on $\left\|u_{n}\right\|_{L^{2}(\mathbb{R})}^{2}$ and martingale inequality (see [16]), we have

$$
\mathbb{E}\left(\sup _{t \in\left[0, T_{0}\right]}\left\|u_{n}\right\|_{L_{x}^{2}}^{2}\right) \leq \mathbb{E}\left(\left\|u_{0, n}\right\|_{L_{x}^{2}}^{2}\right)+C\left\|\Phi_{n}\right\|_{L_{2}^{0,0}}^{2} .
$$

Therefore, the sequence $\left(u_{n}\right)_{n \in \mathbb{N}}$ is bounded and weakly star convergent to a function $u^{*} \in L^{2}\left(\Omega ; L^{\infty}\left(\left[0, T_{0}\right] ; L^{2}(\mathbb{R})\right)\right)$, which satisfies

$$
\mathbb{E}\left(\sup _{t \in\left[0, T_{0}\right]}\left\|u^{*}\right\|_{L_{x}^{2}}^{2}\right) \leq \mathbb{E}\left(\left\|u_{0}\right\|_{L_{x}^{2}}^{2}\right)+C\|\Phi\|_{L_{2}^{0,0}}^{2} .
$$

In the same way as $\mathcal{A}$, define the mapping $\mathcal{A}_{n}$. It is easy to show that $\mathcal{A}_{n}$ is uniformly strict contraction on $\mathfrak{Y}_{r(\omega)}^{t(\omega)}$ in $\mathfrak{X}_{s, b}^{T_{\omega}}$. According to the fixed point theorem, there exists a unique function $u \in \mathfrak{X}_{s, b}^{T_{\omega}}$ such that

$$
u=u^{*}=\lim _{n \rightarrow \infty} u_{n} \quad \text { a.s. in }\left[0, T_{\omega}\right],
$$

where $u_{n}$ is the unique fixed point of $\mathcal{A}_{n}$. Also, we have

$$
\|u(t(\omega))\|_{L^{2}(\mathbb{R})} \leq\left\|u^{*}\right\|_{L^{\infty}\left(\left[0, T_{0}\right] ; L^{2}(\mathbb{R})\right)} .
$$

Thus, we can emerge a solution on $\left[T_{\omega}, 2 T_{\omega}\right]$. Hence, the solution $u$ can be extended to $\left[0, T_{0}\right]$ almost surely by reiteration. This completes the proof of Theorem 2 . 


\section{Summary and discussion}

This paper is devoted to employ the Fourier restriction method, the Banach contraction principle, and some basic inequalities for investigating nonlinear SPDEs and for proving local and global well-posedness results for their solutions in convenient function spaces. Our attention is focused on the stochastic Kawahara Eq. (1), which is a fifth-order shallow water wave equation considered in random environment. We prove that Eq. (1) is locally well-posed for data in $H^{s}(\mathbb{R}), s>-7 / 4$ and its solution can be extended to a global one on $\left[0, T_{0}\right]$. The Fourier restriction method is proposed due to the non-zero singularity of the phase function $\phi$.

The deterministic Kawahara Eq. (7) was discussed by Jia and Huo in [18]. They proved local well-posedness result for data in $H^{s}(\mathbb{R}), s>-7 / 4$. In this paper, we extend their result and handle the stochastic version of the Kawahara equation by choosing new appropriate stochastic function spaces (such as the space $\mathfrak{X}_{s, b}^{T}$ ) and estimating the stochastic convolution (12) in these spaces. That is, we consider a realistic situation of the fifth-order shallow water wave equations. We believe that the ideas which we have suggested in this paper can be also applied to a wide class of stochastic nonlinear evolution equations in the field of mathematical physics, for instance, the stochastic modified Kawahara, generalized $\mathrm{KdV}$, Hirota-Satsuma coupled KdV, and Swada-Kotera equations.

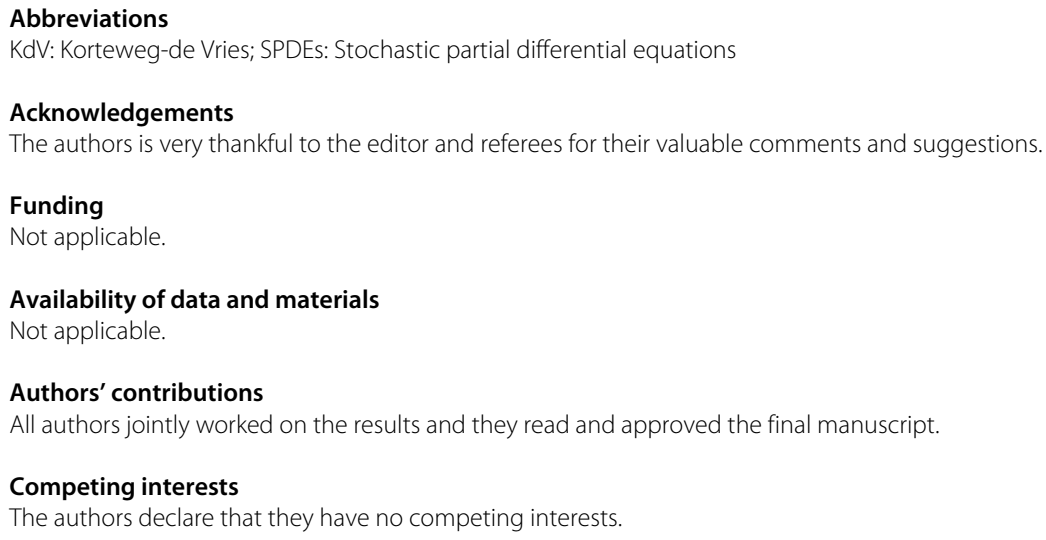

7. Wu, T. Y.: Generation of upstream advancing solitons by moving disturbances. J. Fluid Mech. 184, 75-99 (1987)

8. Ghany, H. A., Hyder, A.: White noise functional solutions for the Wick-type two-dimensional stochastic Zakharov-Kuznetsov equations. Int. Rev. Phys. 6, 153-157 (2012) 
9. Ghany, H. A., Okb El Bab, A. S., Zabal, A. M., Hyder, A.: The fractional coupled KdV equations: exact solutions and white noise functional approach, Vol. 22 (2013)

10. Ghany, H. A., Hyder, A.: Exact solutions for the Wick-type stochastic time-fractional KdV equations. Kuwait J. Sci. 41, 75-84 (2014)

11. Ghany, H. A., Hyder, A.: Abundant solutions of Wick-type stochastic fractional 2D KdV equations, Vol. 23 (2014)

12. Ghany, H. A., Elagan, S. K., Hyder, A.: Exact travelling wave solutions for stochastic fractional Hirota-Satsuma coupled KdV equations. Chin. J. Phys. 53, 1-14 (2015)

13. Ghany, H. A., Hyder, A., Zakarya, M.: Non-Gaussian white noise functional solutions of $\chi$-Wick-type stochastic KdV equations. Appl. Math. Inf. Sci. 11, 915-924 (2017)

14. Hyder, A., Zakarya, M.: Non-Gaussian Wick calculus based on hypercomplex systems. Int. J. Pure Appl. Math. 109, 539-556 (2016)

15. Ghany, H. A., Zakarya, M.: Generalized solutions of Wick-type stochastic KdV-Burgers equations using exp-function method. Int. Rev. Phys. 8, 38-46 (2014)

16. Da Prato, G., Zabczyk, J.: Stochastic equations in infinite dimensions. Cambridge University Press, Cambridge (1992)

17. Huo, Z:: The Cauchy problem for the fifth-order shallow water equation. Acta Math. Appl. Sin. Engl. Ser. 21, 441-454 (2005)

18. Jia, Y., Huo, Z.: Well-posedness for the fifth-order shallow water equations. J. Diff. Equat. 246, 2448-2467 (2009)

19. Tao, S. P., Cui, S. B.: Local and global existence of solutions to initial value problems of nonlinear Kaup-Kupershmidt equations. J. Acta Math. Sin. Engl. Ser. 21, 881-892 (2005)

20. Zhao, X. Q., GU, S. M.: Local solvability of Cauchy problem for Kaup-Kupershmidt equation. J. Math. Res. Exposition. 30, 543-551 (2010)

21. Kenig, C. E., Ponce, G., Vega, L.: A bilinear estimate with applications to the KdV equation. J. Amer. Math. Soc. 9, 573-603 (1996)

22. de Bouard, A., Debussche, A.: On the stochastic Korteweg-de Vries equation. J. Funct. Anal. 154, 215-251 (1998)

23. de Bouard, A.: White noise driven Korteweg-de Vries equation. J. Funct. Anal. 169, 532-558 (1999)

24. Ghany, H. A., Hyder, A.: Local and global well-posedness of stochastic Zakharov- Kuznetsov equation. J. Comput. Anal. Appl. 15, 1332-1343 (2013)

25. Printems, J.: The stochastic Korteweg-de Vries equation in $L^{2}(\mathbb{R})$. J. Diff. Equat. 153, 338-373 (1999)

\section{Submit your manuscript to a SpringerOpen ${ }^{\circ}$ journal and benefit from:}

- Convenient online submission

- Rigorous peer review

- Open access: articles freely available online

- High visibility within the field

- Retaining the copyright to your article

Submit your next manuscript at $\gg$ springeropen.com 